\title{
Analysis of Two Points Grounding Accident in CT Secondary Circuit
}

\section{Li Yuanyi ${ }^{1}$, Li Yueyue ${ }^{2}$, Nie Qixin ${ }^{2}$, Qian Cheng ${ }^{2}$, Tang Hui ${ }^{3}$, Lin Yuan ${ }^{3}$, Zhang Fusheng ${ }^{3}$, Shi Diancai $^{3}$, Liu Ren $^{3}$, Zhao Chuanzong ${ }^{3}$}

${ }^{1}$ State Grid Liaoning Electric Power Co., Ltd. Shenyang power supply company.

${ }^{2}$ State Grid Liaoning Electric Power Co., Ltd. Electric Power Research Institute.

${ }^{3}$ State Grid Liaoning Electric Power Co., Ltd. Fushun power supply company.

Keywords: Current transformer, Relay protection, Impedance

\begin{abstract}
The current transformer (CT) is a kind of transformer. The working principle of CT is briefly introduced. Combined with the actual case of relay error trip accident, the specific cause of the accident by two-point grounding of the secondary circuit loop of the current transformer is further analyzed. There are two points grounding in the B phase circuit in the current transformer. The disturbance of instantaneous potential difference between two points of the ground network causes the protection device to flow into the larger current, thus causing the differential protection device to act. At the same time, the standard requirements for the secondary circuit grounding of the current transformer are put forward. The preventive measures that can be taken in every link of substation operation and maintenance are given.
\end{abstract}

\section{Introduction}

Current transformer (CTA) is a transformer with instrument function. According to the principle of electromagnetic induction, a large current on one side is converted to secondary circuit small current, and the current at low voltage side is measured. CT is composed of closed cores and windings. The number of turns on the primary winding of CT is very small, and the number of turns on the secondary side winding is more. CT is connected in series to the test line. When the current transformer is working, its secondary circuit is always closed. Because the impedance of series coil of measuring instrument and protection loop is very small, the current transformer is close to the short-circuit state when it works. The current transformer is to convert the large current on the primary side to a small current on the secondly side, and the secondly side electric circuit cannot be opened.

In order to ensure the correct work of relay protection and automatic device, there are clear regulations in the "eighteen major anti accident measures of the national Power Grid Corp" and "technical regulations of relay protection and safety automatic device". The secondary circuit of the current transformer must have only one grounding point, which is usually grounded by the terminals of the terminal box. The relay protection and safety automatic device in the substation, because of the construction, overhaul, and reconstruction, there are two grounded points in the secondary circuit loop of the current transformer. Direct or indirect triggering of malfunction of relay protection and automatic safety devices causes circuit breaker tripping. Before the equipment is put into operation, it is necessary to analyze and inspect secondary circuit grounding conditions of relay protection to avoid accidents.

\section{Misoperation accident of relay protection caused by two- point grounding of current transformer}

The secondly loop of the current transformer is mostly outside, with wide distribution and many connection devices. Because of the long running time, the insulation aging or human factors cause two grounding problems, in the secondly circuit of the current transformer. Once the secondly circuit is grounded, it will lead to incorrect operation of the protection device and large power 
failure.

In April 27, 2016, a 220kV substation line protection device started, split phase differential exit, switch trip (reclosing not cast). After the accident, the operator and overhauler in the station inspected the appearance of $1 \#$ main transformer body, high voltage side circuit breaker and current transformer, medium voltage side circuit breaker and current transformer, and 1\# main transformer wire. At the same time, the relay protection device was checked. No abnormalities were found.

\section{Analysis of the cause of the accident}

After the accident, each side protection action recorded wave chart was taken for analysis. The main transformer differential protection misoperation is due to the single phase grounding of the secondary circuit of the B phase current transformer, and the two-point grounding fault. Moreover, the quality of indoor and outdoor equipotential grounding grids in substations is not good, which leads to a grounding fault in a certain $220 \mathrm{kV}$ line. A fault current causes potential difference between the two grounding points. The potential difference forms an additional current in the protection zero sequence winding, and the fault current in the grounding grid is flowing into the B phase circuit of the current transformer, which causes the B phase sampling value of the 1\# main transformer protection device to be large, and the differential current action threshold is finally reached. In the end, the $1 \#$ main transformer protection device was misoperated. The current transformer is decomposed and checked. In the CT secondary circuit junction box, due to the influence of dust and other factors, the protective gap position of the secondary circuit open circuit protection device is intermittent grounding. The substation only laid two equal potential grounding platforms in the relay protection chamber. The grounding grid is not laid in the outdoor cable trench. As a result, there is pressure difference between the two equal potential grounding platform and the outdoor main grounding grid. When the secondary side is grounded, a circulating current is generated between the grounding points.

From the above analysis, it can be seen that when the two side circuit of the current transformer is connected to the ground, on the one hand, the potential difference between the two locations produces a great extra current on the two winding. On the other hand, there is an undue shunt, for example, two connection locations are connected with the earth to form a parallel circuit, and the current winding is short circuited, so that the winding current of the protective device is greatly reduced. The combined effect of the above 2 factors makes the current of the injection protection device differ greatly from the fault current induced by the secondary of the current transformer, causing the incorrect action of the protective device. The protection devices that are seriously affected include current differential protection and zero sequence current protection.

\section{Grounding requirements and two point grounding preventive measures for current transformer's secondary side loop}

There is only one grounding point for the secondary side loop of the current transformer. The grounding point of the current transformer is installed on the grounding copper bar in the outdoor terminal box. The secondary side loop of the common current transformer must be in the relevant protection cabinet screen, and there is one grounding point.

Two point grounding preventive measures for current transformer's secondary side loop

(1) In the capital construction and overhaul technical renovation project, the exact location of the grounding point must be clearly specified on the engineering blueprint.

(2) In the course of construction, the construction is strictly according to the drawings. If the construction personnel have doubts about the grounding position of the secondary side loop, they should contact the designer in time to solve the problem.

(3) Before commissioning, the secondary side loop wiring and Insulation check shall be carried out by the construction technician and fill in the record.

(4) When conducting insulation inspection, it is not appropriate to take spot checks, and should be checked one by one. 
(5) For reconstruction and overhaul parts, the specific location of the existing grounding points should also be identified before construction.

(6) In view of the influence of dust and other factors in the two side connection box, the intermittent grounding occurs, and the operation and maintenance unit should actively contact the current transformer manufacturer, jointly formulate the dismantling scheme of the open circuit protection device. Avoid the secondary side loop grounding of the current transformer due to external environmental interference.

(7) Before the commissioning, the quality inspection and acceptance personnel will carry out the re examination of wiring and insulation, and fill in the records according to the relevant technical requirements. And refer to the record of the builders, ask for a review of the missing and doubtful parts. Timely rectification of defects.

(8) Strictly according to the construction technology, improve the quality of construction. Carry out technical training for construction personnel, and put forward specific secondary side loop grounding process requirements.

(9) Strengthen the acceptance of the construction technology of the new substation. The secondary loop insulation must be checked strictly according to the eighteen counter measures.

\section{Problems that should be paid attention to by operation and maintenance personnel}

During routine maintenance, the maintenance personnel should make full use of the timing of protection check and equipment outage to carry out the secondary loop grounding and Insulation check. For locations where the indoor protective screen and outdoor terminal box have two grounding locations, measures should be taken as soon as possible to keep only one grounding location in the outdoor terminal box. In view of the failure to meet the requirements, timely rectification and elimination of hidden dangers in equipment operation. If the relevant loop changes may be involved, it is important to verify the actual location of the loop location and ensure that it meets the requirements. According to the number of the secondary side winding, the actual input of each winding and the actual location of the loop grounding point, the ledger is established. Combined with actual cases, the training of maintenance personnel is carried out to improve the maintenance level.

\section{Conclusions}

In the power system, the secondary side loop plays a very important role in ensuring the safe operation of the system. In order to ensure the correct work of relay protection and automatic device, the secondary side circuit of the current transformer must have one reliable electrical connection place. Therefore, preventive measures should be taken in all aspects of substation operation to eliminate hidden danger of multi point grounding. To avoid accidents caused by two point grounding of the secondary side loop of current transformer, ensure the safe and stable operation of the power grid and improve the reliability of power supply.

\section{References}

[1] Eighteen major anti accident measures of the national Power Grid Corp (key requirements for relay protection)

[2] Yang Guancheng, principles of power system automation (Third Edition) [M]. Beijing: China Electric Power Press, 2002.

[3] Li Chengzheng, Li Xianyan, application of oil immersed current transformer and research and development of new products, electrical equipment, January 2007, Vol. eighth, first, 29-31.

[4] Zhang Fang, 500kV current transformer explosion accident analysis, power construction, November 2006, volume twenty-seventh, eleventh, 66-67 page. 
[5] Hu Huiran, Zhang Junfeng, 2000 2001 500kV current transformer accident analysis and preventive measures, electrical equipment, December 2002, Vol. third, fourth, 18-22.

[6] Peng Jun, Liu Yong, etc., explosion analysis of 500kV current transformer, Sichuan electric power technology, 2002, fourth issue, page 27-29. 